\title{
Clinical Results of Percutaneous Transluminal Angioplasty and Stenting for Intracranial Vertebrobasilar Atherosclerotic Stenoses and Occlusions
}

\author{
M. TSUURA, T. TERADA*, O. MASUO*, T. TSUMOTO*, H. YAMAGA*, T. ITAKURA*, \\ H. MATSUMOTO**, G. HYOUTANI***, S. HAYASHI \\ Department of Neurological Surgery, Minami Wakayama Medical Center, Wakayama; Japan \\ *Department of Neurological Surgery, Wakayama Medical University, Wakayama; Japan \\ **Department of Neurological Surgery, Naga Public Hospital, Wakayama; Japan \\ ***Department of Neurological Surgery, Japan Red Cross Society Wakayama Medical Center, Wakayama; Japan
}

Key words: PTA/stenting, vertebrobasilar stenosis, complications

\section{Summary}

Eighteen patients with intracranial vertebrobasilar stenosis and occlusion were treated by PTA or stenting. In 11 of 18 cases, only PTA was performed and in seven of 18 cases, we used stents. The mean stenosis before and after PTA/stenting was $82.8 \%$ and $22.3 \%$, respectively. In 11 cases of PTA only, the stenotic rate decreased from $81.8 \%$ to $29.6 \%$, while $85.0 \%$ of the stenotic rate remarkably reduced to $6.0 \%$ in seven cases of stenting.

The 30 days morbidity and 30 days mortality rate were $5.5 \%$ and $5.5 \%$, respectively. There was only one haemorrhagic complication (cerebellar haemorrhage) in cases of stenting, and no ischemic events during or after the procedures. Restenosis (more than 50\% stenosis) occurred in four of 18 cases $(22.2 \%)$ during mean followup period of 12 months. Two patients with VA occlusion before treatment, developed restenosis and reocclusion. Complete total occlusion seems to be a high-risk lesion and strict follow-up is required.

In this study, PTA/stenting for intracranial vertebrobasilar artery stenosis or occlusion is an effective treatment, but strict indications may be required because procedure-related 30 days morbidity rate was $5.5 \%$ in addition to unclear natural history.

\section{Introduction}

To date, intracranial vertebrobasilar atherosclerotic stenosis and occlusion have been generally treated with conservative therapy including administration of the antiplatelet agent. Although natural history of the intracranial vertebrobasilar stenosis is still unkown, several reports shows high stroke risk and high mortality rate of these lesions, especially in cases where the medical treatment failed ${ }^{1,2}$.

In addition, direct surgery (bypass surgery, etc) for intracranial vertebrobasilar stenosis has been reported to have high complication rate of the procedures ${ }^{3}$. Therefore PTA/stenting for intracranial vertebrobasilar atherosclerotic stenosis and occlusion has been performed as an alternative procedure for highrisk patients with intracranial vertebrobasilar stenosis and occlusion. To examine the safety and efficacy of the procedures, we report our clinical results and complications of PTA and stenting for intracranial vertebrobasilar atherosclerotic stenoses and occlusions.

\section{Patients and Methods}

Eighteen patients with intracranial vertebrabasilar stenoses and occlusions (18 lesions) were treated by PTA or stenting. Of 18 pa- 


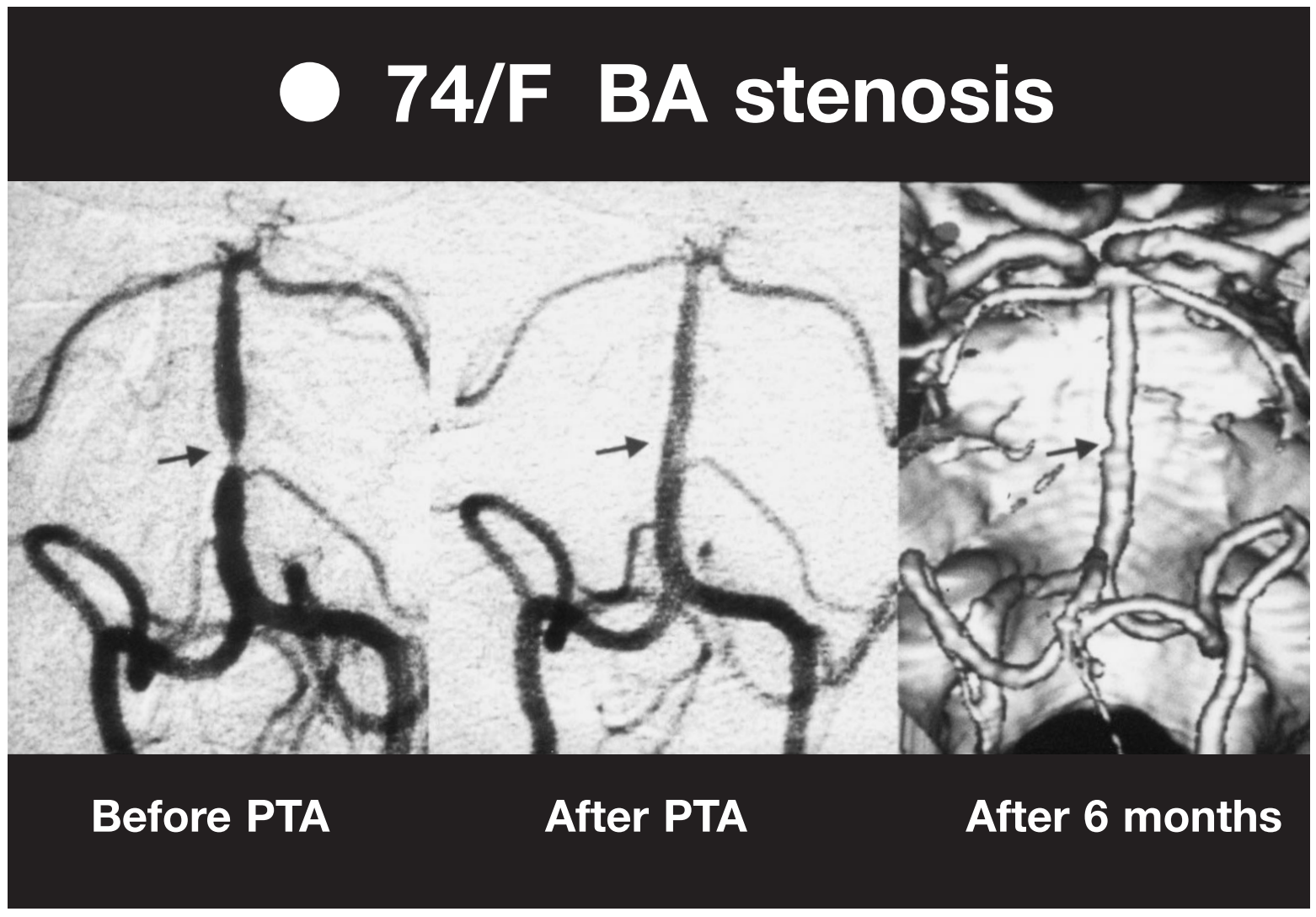

Figure 1 VAG of case 1 before PTA (left), immediately after PTA (middle) and follow-up 3-D CT angiogram (right). The stenosis of the basilar artery reduced from $90 \%$ to $10 \%$ after PTA.

tients, 11 cases underwent only PTA, while seven cases were treated by stenting. In 11 cases of only PTA, four and seven cases had basilar artery (BA) stenosis and vertebral artery (VA) stenosis, respectively.

In seven cases of stenting, two, three and two cases suffered from BA stenosis, VA stenosis and VA occlusion, respectively. In principle, PTA and stenting was planned when the patients met next three conditions:

1) symptoms or signs due to intracranial vertebrobasilar stenosis and occlusion,

2) stenotic rate more than $70 \%$,

3 ) medically refractory symptoms or signs.

PTA and stenting was generally performed by percutaneous transfemoral approach under local anesthesia. In short, the guiding catheter (6-7 Fr) was placed in the vertebral artery as distally as possible and the diameters of the normal artery at the just distal and proximal sites of the stenosis and the length of the steno- sis were measured as precisely as possible. Then we carefully passed a 0.014 " microguidewire across the stenosis under roadmapping and advanced a PTA balloon catheter through the stenosis along the microguidewire.

After the proper positioning of the balloon, the PTA balloon was inflated slowly to avoid overinflation. When the impovement of the stenotic rate was insufficient or an elastic recoil or a dissection occurred, we considerd stent implantation in case that stent delivery system could be navigated to the lesions. Postdilatation was added following the stenting, when residual stenosis remained. Finally, we performed conventional angiography to verify the results and detect the distal embolism, and continued anticoagulation(activated clotting time 250$350 \mathrm{~s})$ for 24 to 48 hours after the procedure. Antiplatelet agents were administrated at least one week before the procedure and continued at least two months after the PTA.

In general, the PTA balloon or the stent needs 
to be inflated to a diameter equal to or less than the diameter of the normal artery and cover the entire stenotic lesions to avoid vessel rupture and dissections. We evaluated angiographic results (\% stenosis, restenosis) and clinical outcome (30 days morbidity, neurological deficits at 30 days, 30 days mortality).

\section{Results}

1) In a total of 18 patients, the mean stenosis before and after PTA/stenting was $82.8 \%$ and $22.3 \%$, respectively. In 11 cases of PTA only, stenotic rate decreased from $81.8 \%$ to $29.6 \%$, while $85.0 \%$ of the stenotic rate remarkably reduced to $6.0 \%$ in seven cases of stenting (table 1).

2) Cerebellar haemorrhage occurred within 24 hours after the procedure in one of 18 cases and this was only one haemorrhagic complication. There were no ischemic events during and after the procedures. Therefore, 30 days morbidity was $5.5 \%$ and neurological deficits at 30 days was also $5.5 \%$ due to the cerebellar haemorrhage.

3) In 18 patients, one patient died of arrhythmia within 30 days after PTA/stenting. It was uncertain that the arrhythmia was related to the procedure. The 30 days mortality was $5.5 \%$.

4) Restenosis (more than $50 \%$ stenosis) occurred in four of 18 cases $(22.2 \%)$ during mean follow-up period of 12 months. Two patients with VA occlusion before the treatment, developed restenosis and reocclusion (table 1).

\section{Representative cases}

\section{Case 1}

A 74-year-old woman with a history of diabetes mellitus suffered from transient vertigo and dizziness. Conventional angiography re-

Table 1 Summary of 18 cases of angioplasty and stenting for intracranial vertebrobasilar atherosclerotic stenosis and occlusion

\begin{tabular}{|c|c|c|c|c|c|c|}
\hline Age/sex & Lesion & Presentation & \multicolumn{2}{|c|}{$\begin{array}{c}\text { \% stenosis } \\
\text { pre } \stackrel{\text { post }}{\longrightarrow}\end{array}$} & Procedure & Complications \\
\hline $61 / \mathrm{M}$ & BA stenosis & VBI & $90 \%$ & $50 \%$ & PTA & $(-)$ \\
\hline $71 / \mathrm{M}$ & BA stenosis & infarct & $80 \%$ & $10 \%$ & PTA & $(-)$ \\
\hline $74 / \mathrm{F}$ & BA stenosis & VBI & $90 \%$ & $10 \%$ & PTA & $(-)$ \\
\hline $61 / \mathrm{F}$ & BA stenosis & infarct & $70 \%$ & $50 \%$ & PTA & $(-)$ \\
\hline $67 / \mathrm{M}$ & BA stenosis & infarct & $90 \%$ & $10 \%$ & Stenting & $(-)$ \\
\hline $57 / \mathrm{F}$ & BA stenosis & infarct & $80 \%$ & $10 \%$ & Stenting & $(-)$ rest \\
\hline $66 / \mathrm{M}$ & VA stenosis & VBI & $80 \%$ & $60 \%$ & PTA & $(-)$ \\
\hline $55 / \mathrm{M}$ & VA stenosis & VBI & $70 \%$ & $0 \%$ & PTA & $(-)$ \\
\hline $57 / \mathrm{M}$ & VA stenosis & infarct & $90 \%$ & $50 \%$ & PTA & $(-)$ rest \\
\hline $62 / \mathrm{M}$ & VA stenosis & TIA & $60 \%$ & $30 \%$ & PTA & $(-)$ \\
\hline $55 / \mathrm{M}$ & VA stenosis & TIA & $70 \%$ & $0 \%$ & PTA & $(-)$ \\
\hline $54 / \mathrm{M}$ & VA stenosis & TIA & $60 \%$ & $0 \%$ & PTA & $(-)$ \\
\hline $60 / \mathrm{M}$ & VA stenosis & VBI & $70 \%$ & $33 \%$ & PTA & $(-)$ \\
\hline $68 / \mathrm{M}$ & VA stenosis & VBI & $70 \%$ & $0 \%$ & Stenting & $(-)$ \\
\hline $77 / \mathrm{F}$ & VA stenosis & VBI & $90 \%$ & $0 \%$ & Stenting & haemorrhage \\
\hline $58 / \mathrm{M}$ & VA stenosis & infarct & $95 \%$ & $10 \%$ & Stenting & $(-)$ \\
\hline $65 / \mathrm{M}$ & VA stenosis & infarct & $100 \%$ & $0 \%$ & Stenting & (-) reoccl. \\
\hline $57 / \mathrm{M}$ & VA stenosis & infarct & $100 \%$ & $40 \%$ & Stenting & (-) rest. \\
\hline
\end{tabular}




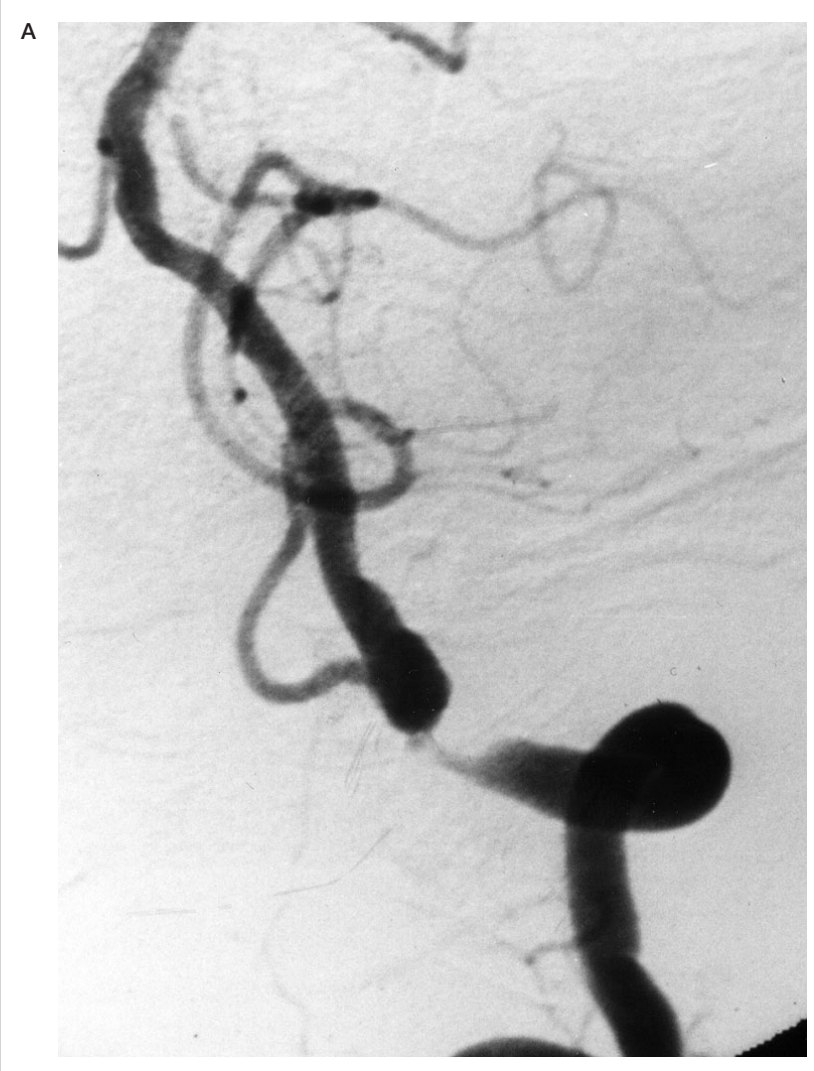

C

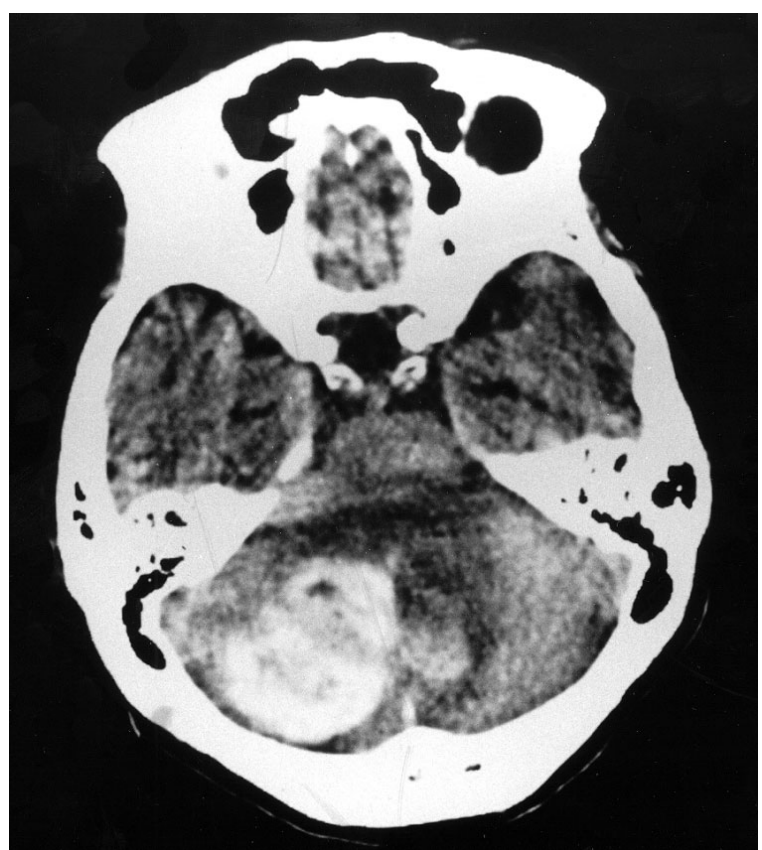

Figure 2 Case 2 A) left VAG showed $90 \%$ stenosis of the left vertebral artery. B) The stenosis disappeared after stent placement. C) CT scan approximately 12 hours after the procedure demonstrated left cerebellar haemorrhage. Surgical removal was required because the patient complained of headache, vomiting and cerebellar ataxia.

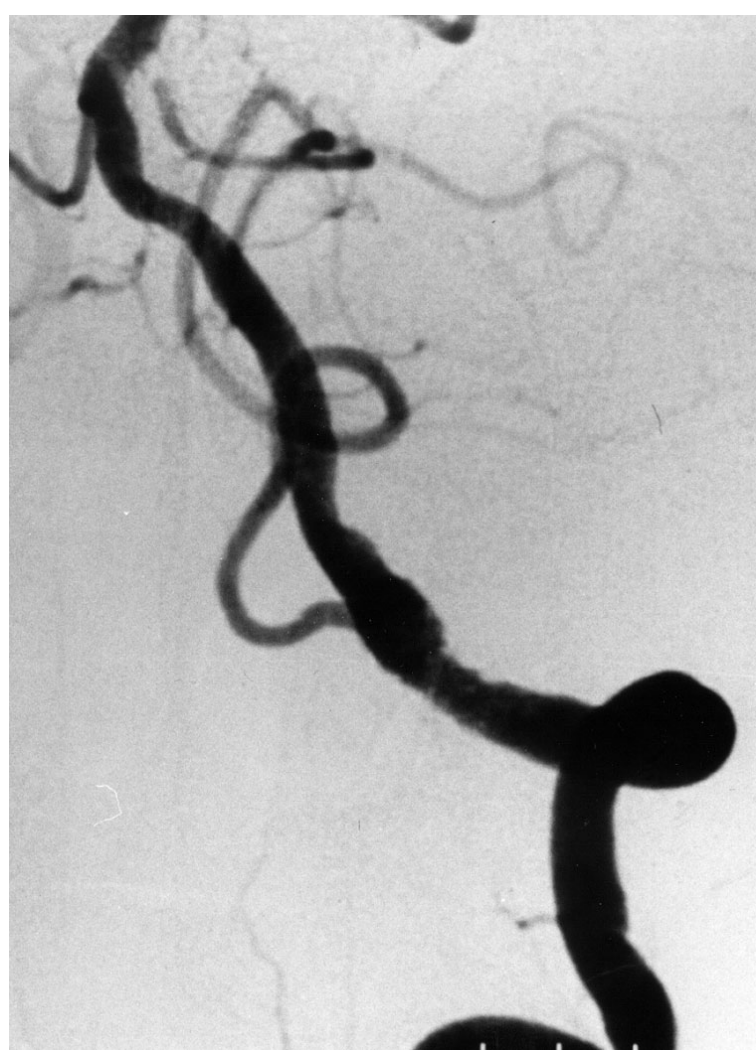

B

vealed a $90 \%$ stenosis of basilar artery and there seemed to be no sufficient collateral circulations. PTA was performed using a Gateway balloon catheter and sufficient improvement of the stenosis $(10 \%)$ was obtained without stenting. She temporary lost conciousness during the inflation of the PTA balloon because of the brainstem ischemia. After six months, the stenosis was still less then $50 \%$ and she did not experience the vertigo and dizziness after the PTA (figure 1).

\section{Case 2}

A 77-year-old woman with a history of hypertension suffered from transient vertigo and dizziness. Conventional angiography revealed a $90 \%$ stenosis of left vertebral artery and right vertebral artery and both posterior communicating arteries were aplastic. We deployed a S670 stent at the site of the stenosis and the stenosis disappeared. She underwent anticoagulation and cerebellar haemorrhage occurred after approximately 12 hours after the procedure. Although surgical removal of the haematoma was performed, she had mild right cerebellar ataxia (figure 2). 


\section{Discussion}

Intracranial vertebrobasilar atherosclerotic stenosis and occlusion have been generally treated with medical treatment such as administration of the antiplatelet agent or anticoagulant. Although natural history of the intracranial vertebrobasilar stenosis is still unkown, several reports demonstrated high stroke risk and high mortality rate of these lesions, especially in medically refractory cases ${ }^{1,2}$. Recently WASID study group showed stroke rate of $10.7 \%$ per 100 patient-years, in particular $15.0 \%$ of BA stenosis and $13.7 \%$ of VA stenosis ${ }^{1}$. Qureshi et $\mathrm{Al}$ also demonstrated that the risk of recurrent stroke was 10.9 per 100 patient-years ${ }^{2}$. In addition to probably poor prognosis, direct surgery such as EC/IC bypass for posterior circulation ischemia was reported to have high complication rate ${ }^{3}$.

Therefore PTA/stenting has been recently expected to become an alternative treatment.

Our results showed marked impovement of the stenotic rate especially in the cases of stenting and good clinical outcome, that is $5.5 \%$ of 30 days morbidity and $5.5 \%$ of 30 days mortality. Since recent reports of PTA or stenting for vertebrobasilar stenosis shows $12-50 \%$ of combined morbidity and mortality rate, $0-20 \%$ of permanent deficits and $0-16 \%$ of mortality, our results seems to be almost same as other authors $^{4-9}$.

In this study, we treated two cases of VA occlusion, which resulted in one restenosis and one reocclusion later. Even if complete recanalization is obtained by stenting, total occlusive lesion is likely to have high risk of late reocclusion. Mori et $\mathrm{Al}$ indicated the following lesions as risk factors of restenosis, dissection and abrupt closure:

1) diffuse (>10 mm),

2) extremely angulated ( $>90$ degrees)

3 ) chronic and totally occluded lesions ${ }^{10}$.

Besides Mehran et $\mathrm{Al}$ demonstrated the following risk factors of in-stent restenosis after coronary stenting:

1) diffuse proliferative and totally occluded lesions,

2) diabetes

3) previous episodes of recurrent in-stent restenosis $^{11}$. Our experience also suggested arterial occlusion is the high-risk lesion of recurrence and strict follow-up should be required.

\section{Conclusions}

PTA/stenting for intracranial vertebrobasilar artery stenosis or occlusion is an effective treatment, but strict indications may be required because procedure-related 30 days morbidity rate was $5.5 \%$ and 30 days mortality rate was $5.5 \%$ in this study.

The stent is useful for sufficient initial gain of the lumen and prevention of elastic recoil or dissection, despite of difficult endovascular access. Complete total occlusion seems to be a high-risk lesion, and strict follow-up should be required.

\section{References}

1 The Warfarin-Aspirin Symptomatic Intracranial Disease (WASID) Study Group: Prognosis of patients with symptomatic vertebral or basilar artery stenosis. Stroke 29: 1389-1392, 1998.

2 Qureshi AI, Suri MFK et Al: Stroke-free survival and its determinants in patients with symptomatic vertebrobasilar stenosis: a multicenter study. Neurosurgery 52: 1033-1040, 2003.

3 Hopkins LN, Budny JL et Al: Complications of intracranial bypass for vertebrobasilar insufficiency. J Neurosurg 70: 207-211, 1989.

4 Nahser HC, Henkes H et Al: Intracranial vertebrobasilar stenosis: angioplasty and follow-up. Am J Neuroradiol 21: 1293-1301, 2000.

5 Rasmussen PA, Perl II J et Al: Stent-assisted angioplasty of intracranial vertebrobasilar atherosclerosis: an initial experience. J Neurosurg 92: 771-778, 2000.

6 Gomez CR, Mirsa VK et Al: Elective stenting of symptomatic basilar artery stenosis. Stroke 31: 95-99, 2000.

7 Levy EI, Horowitz MB et Al: Transluminal stent-assisted angioplasty of the intracranial vertebrobasilar system for medically refractory, posterior circulation ischemia: early results. Neurosurgery 48: 1215-1223, 2001.

8 Levy EI, Hanel RA et Al: Staged stent-assisted angioplasty for symptomatic intracranial vertebrobasilar artery stenosis. J Neurosurg 97: 1294-1301, 2002.

9 Gress DR, Smith WS et Al: Angioplasty for intracranial symptomatic vertebrobasilar ischemia. Neurosurgery 51:23-29, 2002.

10 Mori T, Kazita K et Al: Cerebral angioplasty and stenting for intracranial vertebral atherosclerotic stenosis. Am J Neuroradiol 20: 787-789, 1999.

11 Mehran R, Dangas G et Al: Angiographic patterns of in-stent restenosis Classification and implications for long-term outcome. Circulation 100: 1872-1878, 1999.

M. Tsuura, M.D.

Department of Neurological Surgery Minami Wakayama Medical Center Japan 